\title{
Remodeling of the human endometrial epithelium is regulated by laminin and type IV collagen
}

\author{
TETSUJI TANAKA, CHUNLIAN WANG and NAOHIKO UMESAKI \\ Department of Obstetrics and Gynecology, Wakayama Medical University, \\ Kimi-idera 811-1, Wakayama 641-0012, Japan
}

Received July 15, 2008; Accepted September 24, 2008

DOI: 10.3892/ijmm_00000114

\begin{abstract}
We investigated the expression and function of laminin and type IV collagen (COL-4) in the human endometrial epithelium throughout the menstrual cycle. Their expression was demonstrated by immunohistochemistry, while cytological analysis of the human endometrial epithelial cell line HHUA cultured on laminin- or COL-4-coated plates provided information on their roles in cell proliferation, structure and apoptosis. Laminin and COL-4 were detected in subepithelial basement membrane-like structures and their expression levels varied throughout the menstrual cycle. Laminin expression was significantly decreased in secretory phase endometrial surface epithelium, while COL-4 expression was significantly decreased in late proliferative phase endometrial epithelium and in vascular endothelium. The morphology of proliferating HHUA cells varied depending on the extracellular matrix component coated on the culture plates. COL-4 strongly inhibited cell proliferation of HHUA cells and enhanced Fas antigen (CD95)-mediated apoptosis, while laminin inhibited Fas-mediated apoptosis. These results indicate that the cyclic expression of laminin and COL-4 in basement membrane-like structures of endometrial epithelium may regulate the endometrial epithelial remodeling and embryo implantation during the menstrual cycle.
\end{abstract}

\section{Introduction}

Human endometrial tissues are remodeled every month. Immediately after menstruation, human endometrial cells begin to proliferate, mainly under ovarian estrogen stimulation. After ovulation, the regenerated endometrial tissue differentiates under the influence of ovarian progesterone and estrogen, in preparation for embryo implantation. If no embryo implants in the endometrium, the levels of ovarian estrogen and

Correspondence to: Dr Tetsuji Tanaka, Department of Obstetrics and Gynecology, Wakayama Medical University, Kimi-idera 811-1, Wakayama 641-0012, Japan

E-mail: obgywmu@wakayama-med.ac.jp

Key words: laminin, type IV collagen, endometrial remodeling, apoptosis, Fas, basement membrane progesterone decrease rapidly and induce endometrial shedding, i.e. menstruation. Remodeling of the functional human endometrium must involve cyclic production of extracellular matrix (ECM), as well as cell regeneration and proliferation. Laminin and type IV collagen (COL-4) are well-known major components of epithelial basement membranes in the human body and have been reported to localize in the basement membrane-like structures (BM-like-S) of human endometrial epithelium $(1,2)$. Several studies have shown that both these ECM components are highly expressed in human endometrial stromal cells and deciduas (3-5). Laminin has been reported to inhibit endometrial stromal differentiation $(6,7)$, while both laminin and COL-4 have been found to regulate trophoblast proliferation and differentiation during pregnancy (8-11). Furthermore, abnormal expression of these ECM components in the endometrium was reported in a patient with unexplained infertility (1). We recently demonstrated that laminin and COL-4 regulated normal human endometrial stromal remodeling in an autocrine/paracrine fashion during the menstrual cycle (5). Therefore, in this study, we further investigated the effects of laminin and COL-4 on endometrial epithelial remodeling.

The cyclic changes in the expression levels of laminin and COL-4 in BM-like-S during the menstrual cycle are not fully understood $(1,2)$. Cyclic remodeling of the human endometrium must be regulated not only by cyclic proliferation, differentiation, morphological changes and cell death of endometrial epithelial cells, but also by cyclic regeneration of BM-like-S during the menstrual cycle. However, the roles of laminin and COL-4 in these cyclic functional and morphological changes in the endometrial epithelium have been little studied. Many previous studies have shown that integrin-mediated cell adhesion affects cell viability and proliferation (12-15). Therefore, in the present study, we investigated the expression levels of laminin and COL-4 in BM-like-S, in addition to the effects of these components on cell proliferation, apoptosis and cell colony structure in a human endometrial epithelial cell line that retains many of the intracellular signaling pathways found in normal endometrial epithelial cells.

\section{Materials and methods}

Immunohistochemistry. After receiving written informed consent, normal human endometrial tissues were obtained from 22 patients (aged 25-49 years) with normal menstrual 
cycles whose uteri were surgically removed due to cervical cancer or leiomyoma and from 4 postmenopausal women (aged 51-55 years) with cervical cancer. The tissue samples were fixed in $10 \%$ formalin solution and embedded in paraffin. Serial sections $(3-\mu \mathrm{m}$ thick) were cut and mounted on gelatin-coated glass slides. To confirm the endometrial dating, each sample was stained with hematoxylin and eosin. Tissues were classified into five menstrual stages according to the patients' chart records and histopathological findings: midproliferative period (MP; within 9 days after initiation of menstruation; $n=5$ ); late proliferative period (LP; from 10 days after initiation of menstruation to ovulation; $n=6$ ); midsecretory period [MS; postovulatory period with some decidualization $(<5 \%) ; \mathrm{n}=5$ ]; late secretory period (LS; endometrial tissues with a large amount of decidualization; $\mathrm{n}=6$ ); and postmenopausal period (PM; at least 2 years after spontaneous menopause; $n=4)$.

Immunohistochemical examinations were carried out using an avidin-biotin peroxidase complex technique. Briefly, deparaffinized sections were washed three times with phosphate-buffered saline (PBS) for $5 \mathrm{~min}$ at room temperature. After microwaving, the sections were treated with $0.3 \%$ hydrogen peroxide in absolute methanol to block endogenous peroxidase activity and washed five times with PBS at room temperature. After blocking with normal horse serum (Dako, Carpinteria, CA) (1:20 dilution) for COL-4 staining or normal swine serum (Dako) (1:20 dilution) for laminin staining, the sections were incubated with mouse anti-human type IV collagen IgG (Dako) (1:300 dilution) or rabbit anti-human laminin $\operatorname{IgG}$ (Dako) (1:600 dilution) overnight at $4^{\circ} \mathrm{C}$ and then washed five times with PBS. The sections were then incubated with biotinylated horse antimouse IgG (Dako) (for COL-4) or biotinylated swine antirabbit IgG (Dako) (for laminin) for $30 \mathrm{~min}$ at room temperature, followed by incubation with avidin-biotinylated horseradish peroxidase complexes (Vector Laboratories Inc., Peterborough, UK) for $60 \mathrm{~min}$ at room temperature. After five washes with $0.05 \mathrm{M}$ Tris-buffered saline, $\mathrm{pH} 7.4$, positive reactions were visualized with $0.03 \%$ hydrogen peroxide and $0.1 \%$ 3,3-diaminobenzidine-tetrahydrochloride (DAB) (Dako) in Tris buffer. After another five washes, the sections were counterstained with Mayer's hematoxylin (Nakalai, Kyoto, Japan). To test the specificity of the immunolabeling, negative control sections from each sample were stained by the same protocol without the first antibody. Expression of laminin and COL-4 in endometrial BM-like-S was semiquantitatively evaluated by scoring immunoreactive intensity (score 0 , negative; score 1 , very weakly positive or scattered positive; score 2 , positive and score 3 , strongly positive). For each section, the immunoreactivities were scored in three BM-like-S of the endometrium: the BM-like-S at the luminal surface epithelium, the BM-like-S at the glandular epithelium and the BM-like-S under the vascular endothelium. Data were plotted graphically as means $\pm \mathrm{SD}$ and data comparisons were analyzed by $\mathrm{t}$-tests $(\mathrm{n}=4-6)$. Values of $\mathrm{p}<0.05$ were considered to indicate statistically significant differences.

Cell line and cell culture. The human endometrial epithelial cell line, HHUA, was obtained from Riken Cell Bank (Tsukuba, Japan). The cells were cultured in OPTI-MEM
(Invitrogen, Carlsbad, CA, USA), 5\% fetal calf serum (FCS) (Equitech Bio Inc., Ingram, TX, USA), penicillin (PC; $100 \mathrm{U} / \mathrm{ml})$ (Invitrogen) and streptomycin (SM; $100 \mu \mathrm{g} / \mathrm{ml})$ (Invitrogen). The HHUA cell line was selected for this study because the cells express high levels of functional Fas antigen (16), as well as functional estrogen and progesterone receptors (17), as in normal human endometrial epithelium. The cells also form glandular luminal structures in collagen gel cultures, similar to the structures formed by normal glandular epithelial cells (18). Karyotype analysis of 20 HHUA cells indicated normal 46XX karyotype (data not shown). Based on these characteristics, HHUA cells are considered to retain many of the intracellular signaling pathways found in normal endometrial epithelial cells.

Cell viability assay. Cell viability was assayed using a cell proliferation assay kit (Dojin, Tokyo, Japan). The stimulatory effect of murine anti-human Fas IgM (clone CH-11; MBL, Nogoya, Japan) on the growth of HHUA cells was determined as follows: on day 1, HHUA cells in the log phase were detached using $0.25 \%$ trypsin/1 mM EDTA (Invitrogen) and cultured overnight in $96-$ well culture plates $(5,000$ cells/well $)$ containing OPTI-MEM/5\% FCS/PC/SM. On day 2, various concentrations of anti-Fas $\operatorname{IgM}$ were added to the cells. On day 4 , viable cell numbers were counted.

The effects of ECM components on the numbers of viable cells were examined by culturing HHUA cells on type I collagen-, COL-4-, fibronectin- or laminin-coated 96-well plates (all from Becton-Dickinson, Franklin Lakes, NJ). Noncoated 96-well plates purchased from Nalge Nunc International (Copenhagen, Denmark) were used for negative control cultures.

All experiments were performed in triplicate. Graphical data were plotted as means \pm SD and data comparisons were analyzed by t-tests $(n=12)$ and/or one-way ANOVA. Values of $\mathrm{p}<0.05$ were considered to indicate statistically significant differences.

Semi-quantitative flow cytometry. HHUA cells were detached from tissue culture dishes using $3 \mathrm{mM}$ EDTA in PBS and stained according to the following procedure: Cells $\left(3 \times 10^{5}\right)$ were incubated with an excess of one of the primary antibodies for $20 \mathrm{~min}$ at $4^{\circ} \mathrm{C}$ and then washed twice with washing buffer (PBS containing 2\% FCS and $0.1 \% \mathrm{NaN}_{3}$ ). The cells were then reacted with FITC-conjugated goat anti-mouse IgG $(\mathrm{H}+\mathrm{L})\left(\right.$ Dako) as a secondary antibody for $20 \mathrm{~min}$ at $4^{\circ} \mathrm{C}$ and washed twice. Finally, the cells were suspended in $200 \mu \mathrm{l}$ of washing buffer and analyzed using a FACSCalibur flow cytometer (Becton-Dickinson). The primary antibodies used were: mouse anti-human Fas (CD95) monoclonal antibody (clone UB2; MBL); mouse anti-human CD29 monoclonal antibody (clone TDM29; Cymbus Biotech Ltd., Hampshire, UK); mouse anti-human CD49a monoclonal antibody (clone TS2/7; Serotec Ltd., Oxford, UK); mouse anti-human CD49b monoclonal antibody (clone 31H4; Serotec Ltd.); mouse anti-human CD49c monoclonal antibody (clone 11G5; Cymbus Biotech Ltd., Chandlers Ford, UK); mouse antihuman CD49d monoclonal antibody (clone 44H6; Cymbus Biotech Ltd.); mouse anti-human CD49e monoclonal antibody (clone SAM1; Beckman Coulter Japan, Tokyo, Japan); and 

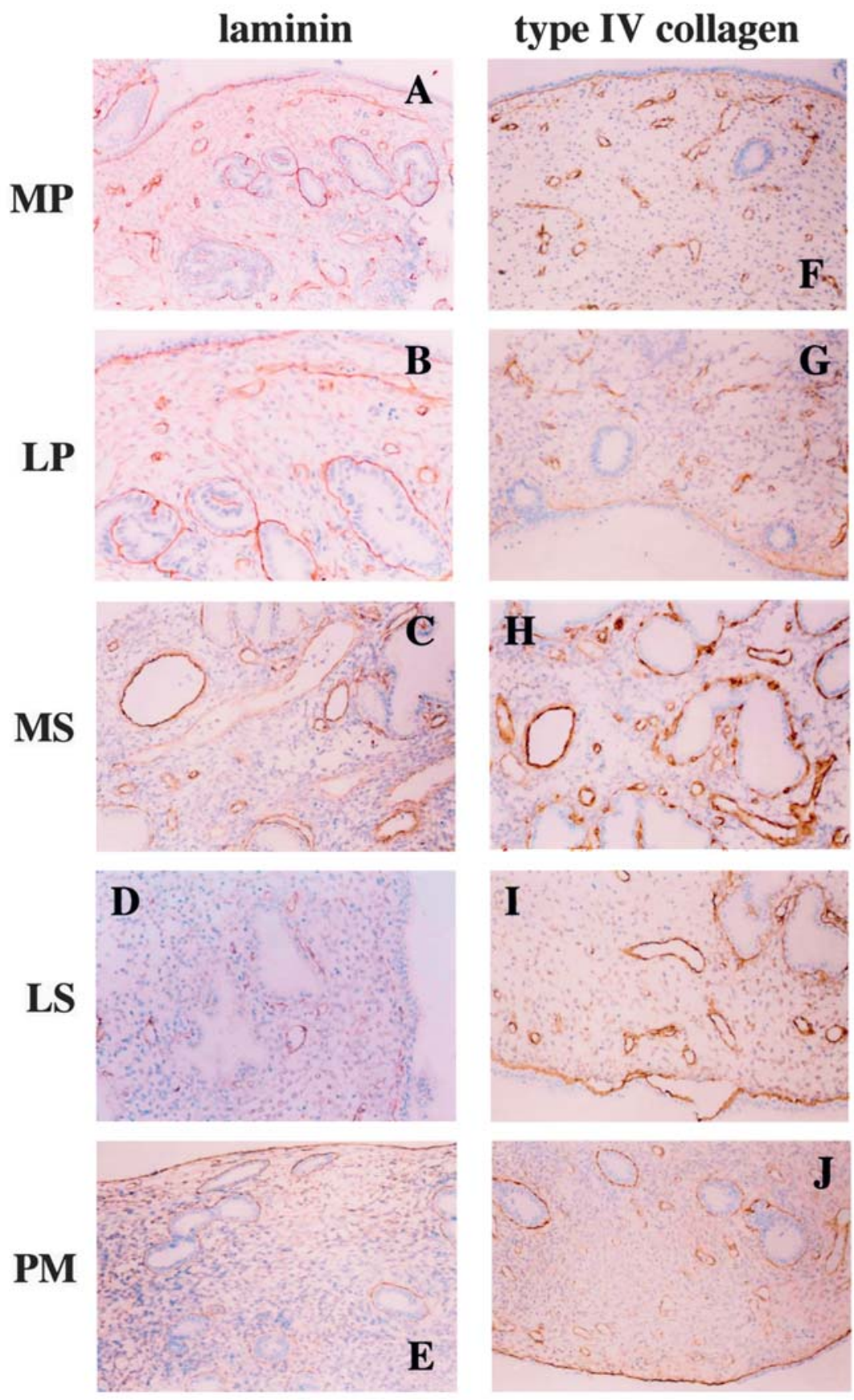

Figure 1. Expression of laminin and COL-4 in human endometrial epithelial tissues. The endometrial tissues were classified into the following groups: MP, mid-proliferative period; LP, late proliferative period; MS, mid-secretory period; LS, late secretory period and PM, postmenopausal endometrium. (A-E) Laminin expression. (F-J) COL-4 expression.

mouse anti-human CD49f monoclonal antibody (clone 4F10; Cymbus Biotech Ltd.). HHUA cells treated with the secondary antibody alone were used as negative controls.

DNA fragmentation assay. HHUA cells in the log phase were detached with $0.25 \%$ trypsin/ $1 \mathrm{mM}$ EDTA and then cultured overnight in the ECM-coated culture dishes $\left(3 \times 10^{6}\right.$ cells/ dish) containing OPTI-MEM/5\% FCS/PC/SM. On day 3, murine anti-human Fas IgM (clone $\mathrm{CH}-11$ ) (final concentration, $20 \mathrm{ng} / \mathrm{ml}$ ) was added to the cells. On day 4, genomic DNA was extracted from all cells, including the dead ones, using a SepaGene DNA extraction kit (Sankyo-Junyaku Co. Ltd., Tokyo, Japan) and treated with $100 \mu \mathrm{g} / \mathrm{ml}$ of RNase A
(Sigma, St. Louis, MO, USA) in TE buffer (10 mM Tris, $\mathrm{pH} 8.0,2 \mathrm{mM}$ EDTA) for $90 \mathrm{~min}$ at $37^{\circ} \mathrm{C}$ to remove any contaminating RNA. Next, $20 \mu \mathrm{g}$ of the genomic DNA was electrophoresed in a $1.2 \%$ agarose gel at $50 \mathrm{~V}$ for $\sim 2 \mathrm{~h}$, stained with $5 \mu \mathrm{g} / \mathrm{ml}$ of ethidium bromide and visualized by UV fluorescence.

\section{Results}

Expression of laminin and COL-4 in BM-like-S of normal human endometrial epithelium. Normal human endometrial tissues were analyzed for expression of laminin and COL-4 by immunohistochemistry (Fig. 1). Endometrial stromal cells 
A

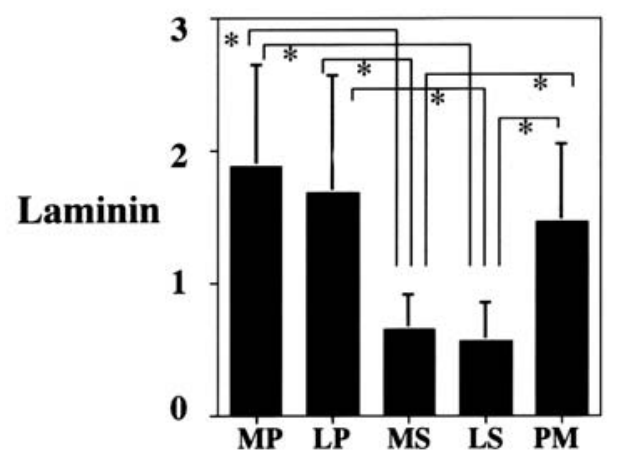

D

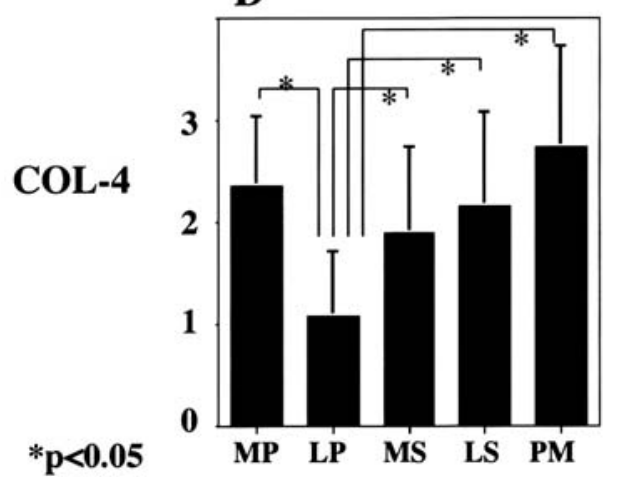

B

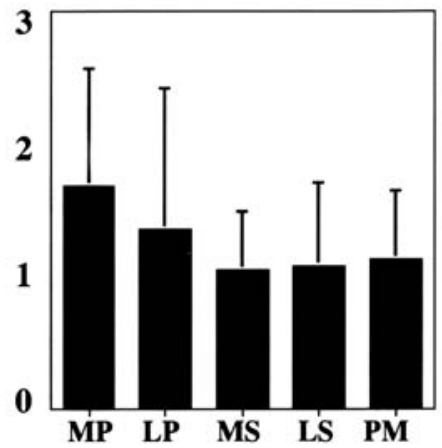

$\mathbf{E}$

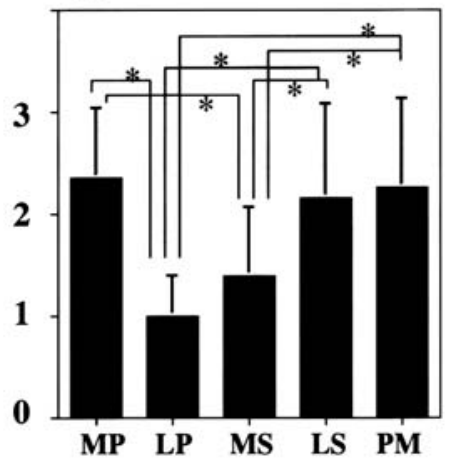

C

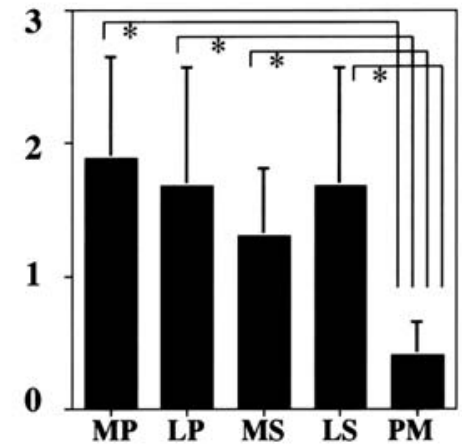

Figure 2. Expression of laminin and COL-4 in BM-like-S of endometrium. Expression levels of both ECM components in BM-like-S were semi-quantitatively scored according to the criteria described in Materials and methods. (A and D) BM-like-S at luminal surface epithelium; (B and E) BM-like-S at glandular epithelium; ( $\mathrm{C}$ and F) vascular endothelium. The endometrial tissues were classified into the following five groups: MP, mid-proliferative period; LP, late proliferative period; MS, mid-secretory period; LS, late secretory period and PM, postmenopausal endometrium. ${ }^{*}$ Indicates a significant difference (p<0.05).

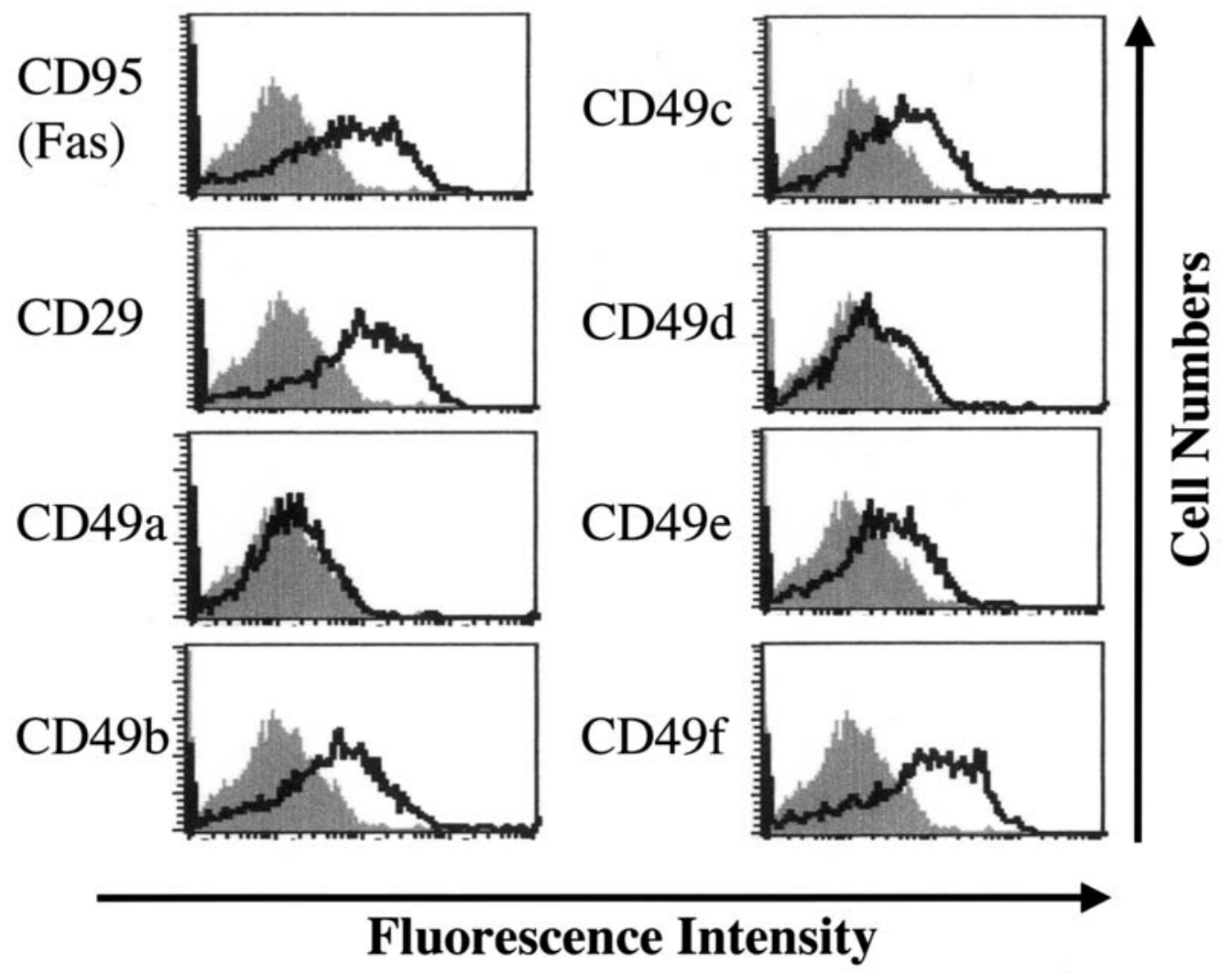

Figure 3. Expression of integrin subunits, receptors for ECM-components, in HHUA cells. Semi-quantitative flow cytometric analysis was performed as described in Materials and methods. The gray figures are negative controls and the thick solid lines show integrin expression. 
A
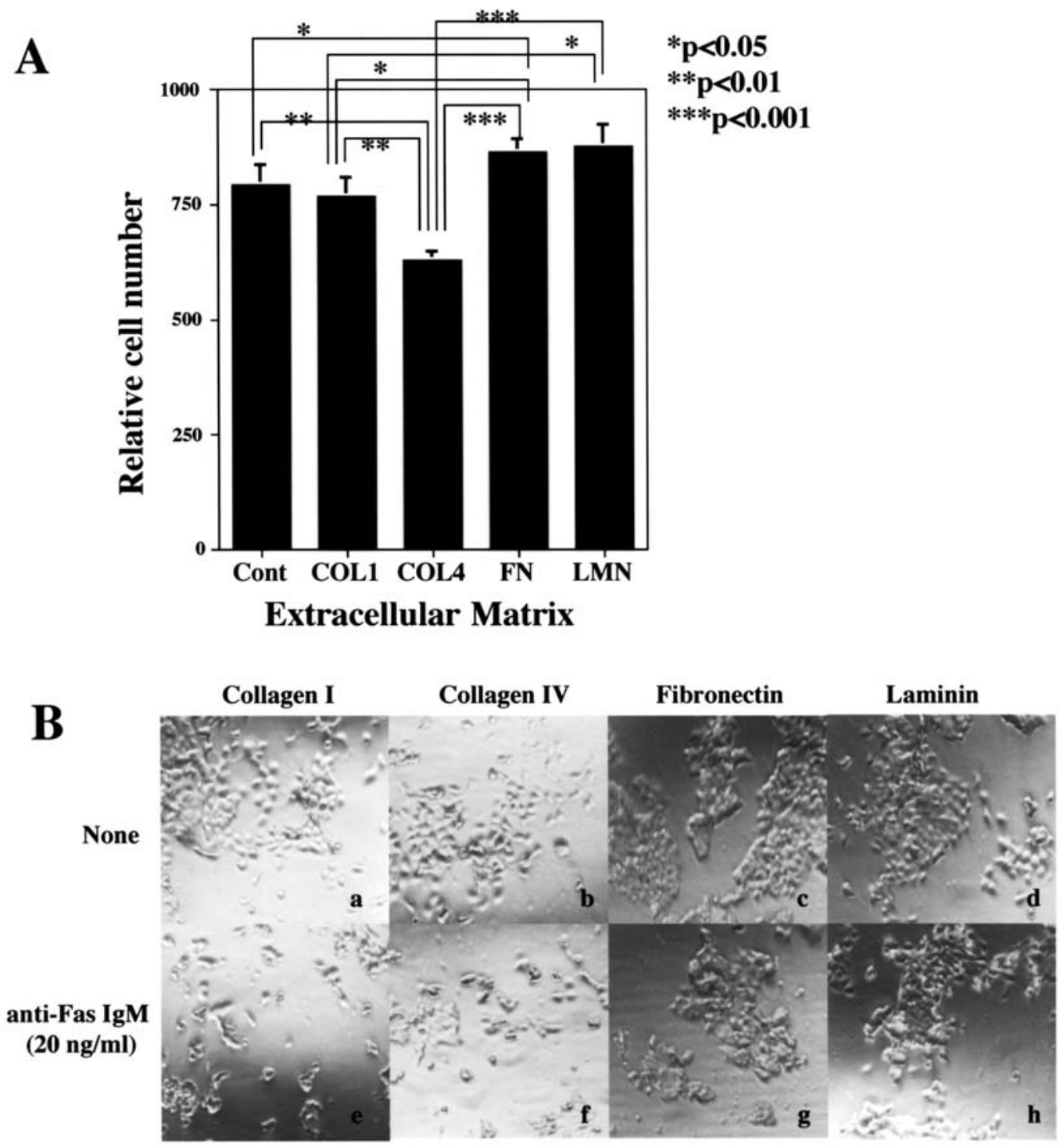

Figure 4. Cell proliferation and microscopic findings of growing cell colonies of HHUA cells cultured on ECM component-coated dishes. (A) Cell proliferation assay of HHUA cells cultured on ECM component-coated dishes. Cont, cells cultured in culture plates from Nalge Nunc International; COL1, cells cultured on type I collagen-coated plates; COL4, cells cultured on COL-4-coated plates; FN, cells cultured on fibronectin-coated plates; LMN, cells cultured on laminin-coated plates. "Indicates significant differences $\left({ }^{*} \mathrm{p}<0.05,{ }^{* * *} \mathrm{p}<0.01\right.$ and $\left.{ }^{* * *} \mathrm{p}<0.001\right)$. (B) The effects of four ECM components on the cell sheet structure of HHUA cells were compared. (a-d) Unstimulated cells; (e-h) anti-Fas IgM-stimulated cells ( $20 \mathrm{ng} / \mathrm{ml}$, overnight).

stained positively for both laminin and COL-4, while endometrial epithelial cells showed no expression of either. However, strong immunoreactivities for both laminin and COL-4 were found in BM-like-S at the endometrial epithelium and vascular endothelium. The immunoreactivities in the $\mathrm{BM}$-like-S were scored according to the criteria described in Materials and methods. As shown in Fig. 2A-C, laminin expression was significantly decreased during the secretory phase in BM-like-S at the luminal surface epithelium, but not at the glandular epithelium or endometrial vascular endothelium. Laminin expression was significantly reduced in BM-like-S at the vascular endothelium in postmenopausal tissues (Fig. 2C). COL-4 expression, however, was significantly decreased in BM-like-S at the luminal surface epithelium (Fig. 2D), glandular epithelium (Fig. 2E) and vascular endothelium (Fig. $2 \mathrm{~F}$ ) during the late proliferative phase.
Expression of integrins in HHUA cells. Integrins are receptors for ECM components. Flow cytometric analyses were performed to examine the expression of integrin subunits by HHUA cells (Fig. 3). HHUA cells expressed CD29 and CD49b-CD49f molecules on the cell surface, with especially high levels of CD29, CD49b, CD49c and CD49f.

Cell growth of HHUA cells cultured on ECM componentcoated plates. If the integrins on HHUA cells bind to specific ECM components, cultured cells should demonstrate morphological differences depending on their interactions with individual ECM components. We therefore examined the effects of laminin and COL-4 on the cell proliferation and morphological changes of HHUA cells cultured on various ECM component-coated dishes. As shown in Fig. 4A, proliferation was strongly inhibited in HHUA cells cultured on COL-4-coated plates, while growth of cells cultured on 
laminin- or fibronectin-coated plates was significantly increased, compared with those cultured on type 1 collagen- or COL-4-coated plates.

There were also clear microscopic differences in the structures of HHUA cell colonies cultured on the different ECM components (Fig. 4Ba-Bd). Scattered HHUA cells proliferated on type I and COL-4-coated dishes (Fig. 4Ba and $\mathrm{Bb})$, while clusters of HHUA cells proliferated on fibronectinand laminin-coated dishes (Fig. 4Bc and Bd).

Modifications of Fas-mediated signals in HHUA cells cultured on individual ECM components. As reported before, anti-Fas $\operatorname{IgM}$ is a strong inducer of growth suppression and DNA fragmentation in HHUA cells (12). We therefore examined the effects of laminin and COL-4 on cell growth inhibition and DNA fragmentation induced by anti-Fas IgM stimulation of HHUA cells cultured on various ECM component-coated dishes. As shown in Fig. 5, laminin prevented cell growth inhibition (Fig. 5A) and DNA fragmentation (Fig. 5B) induced by anti-Fas IgM. However, in HHUA cells cultured on COL-4coated plates, cell growth inhibition (Fig. 5C) and DNA fragmentation (Fig. 5D) induced by anti-Fas IgM, were significantly enhanced.

\section{Discussion}

The present study has demonstrated that specific adhesion between ECM components and endometrial epithelial cells can regulate the viability and proliferation of the endometrial epithelium. We have also shown that the expression of COL-4 and laminin in endometrial BM-like-S changes cyclically during the menstrual cycle.

Recent immunohistochemical studies revealed that human endometrial stromal cells, but not epithelial cells, were immunopositive for COL-4 and laminin, suggesting that both COL-4 and laminin are produced by stromal cells in the human endometrium, but not by epithelial cells and that they are then transported to intercellular spaces and subepithelial BM-like-S (5). The menstrual cycle-dependent production of laminin and COL-4 in human endometrial stromal cells suggests that their cyclic production may be regulated by ovarian steroid hormones, such as estrogen and progesterone. However, the expression levels of laminin and COL-4 in endometrial epithelial BM-like-S vary significantly over a short period of time. Laminin expression was significantly decreased in BMlike-S during the secretory phase, but only at the luminal surface epithelium, not at the glandular epithelium or endometrial vascular endothelium. Moreover, laminin expression was significantly reduced in BM-like-S at the postmenopausal vascular endothelium but not at the postmenopausal endometrial epithelium. These results suggest that the laminin-specific proteolytic activities of secretory phase luminal surface endometrial epithelium and postmenopausal vascular endothelial regions may be rapidly increased. However, these laminin-specific proteolytic activities may not be regulated by ovarian steroid hormones, because no significant changes in laminin expression were observed in BM-like-S of the glandular epithelium or endometrial vascular endothelium during the secretory phase, and because no significant changes in laminin expression were observed in BM-like-S of postmenopausal endometrial epithelium. COL-4 expression, however, was significantly decreased in BM-like-S at the luminal surface epithelium, glandular epithelium and vascular endothelium during the late proliferative phase. These results indicate that the COL-4specific proteolytic activities may be regulated partially by ovarian steroid hormones.

The effects of laminin and COL-4 were investigated by using the human endometrial epithelial cell line, HHUA, which retains many of the intracellular signaling pathways found in normal endometrial epithelial cells. Our flow cytometric analyses revealed that HHUA cells expressed cell surface integrins, which act as receptors for ECM components. HHUA cell growth on COL-4-coated plates was significantly inhibited, and the cell colony structure varied depending on the ECM component coated on the culture dish. These results indicate that cell surface integrins on HHUA cells act as specific functional receptors for ECM components and that the cell proliferation and structure of the human endometrial epithelium can be regulated by ECM components located in BM-like-S, depending on the phase of the menstrual cycle.

We have demonstrated in the present study that laminin inhibits, while COL-4 enhances, Fas-mediated apoptosis of HHUA cells. These results indicate that menstrual cycledependent Fas-mediated apoptotic susceptibility of human endometrial epithelium may be regulated by ECM components in BM-like-S. Our recent study showed that laminin and COL-4 differentially regulated cell viability of normal human endometrial stromal cells (5). The Fas-stimulation of normal human endometrial stromal cells has been reported to stimulate cell viability (19) and interaction with COL-4 has been reported to protect lens epithelial cells from Fas-dependent apoptosis by stimulating the production of soluble survival factors (20). The stimulation of integrin $\alpha 2 \beta 1$ with ECM was shown to inhibit Fas-mediated apoptosis in T lymphocytes by protein phosphatase 2A-dependent activation of the mitogenactivated protein kinase (MAPK)/ERK pathway (21). In osteoblasts, laminin has been reported to enhance Fas-mediated apoptosis via the H-Ras/MAPK pathway (22). Other studies have shown that ligand-bound $B 1$ integrins influenced cell adhesion-mediated drug and radiation resistance in human leukemia cells through inhibition of procaspase-8 (23). The results of current and previous studies suggest that the interaction of cells and ECM-components can inhibit or enhance Fas-mediated apoptosis, dependent on the cell lineage. To our knowledge, the present study is the first to show that laminin inhibits Fas-mediated apoptosis in human endometrial epithelial cells, while COL-4 enhances the process. The mechanisms of laminin- and COL-4-stimulated modification of Fas-mediated apoptosis in HHUA cells has yet to be clarified. We suggest two possibilities: The specific interactions between the cells and ECM components affect Fas-mediated apoptotic signals, or the specific interactions change the cell growth structure, so as to alter the distribution of Fas antigens on the cell surface enough to modify the reactivity of the cells with anti-Fas IgM and Fas ligand.

In this study, we have demonstrated two important facts: that expression of laminin and COL-4 in BM-like-S of human endometrial epithelium varies during the menstrual cycle and that interaction between human endometrial epithelial cells 

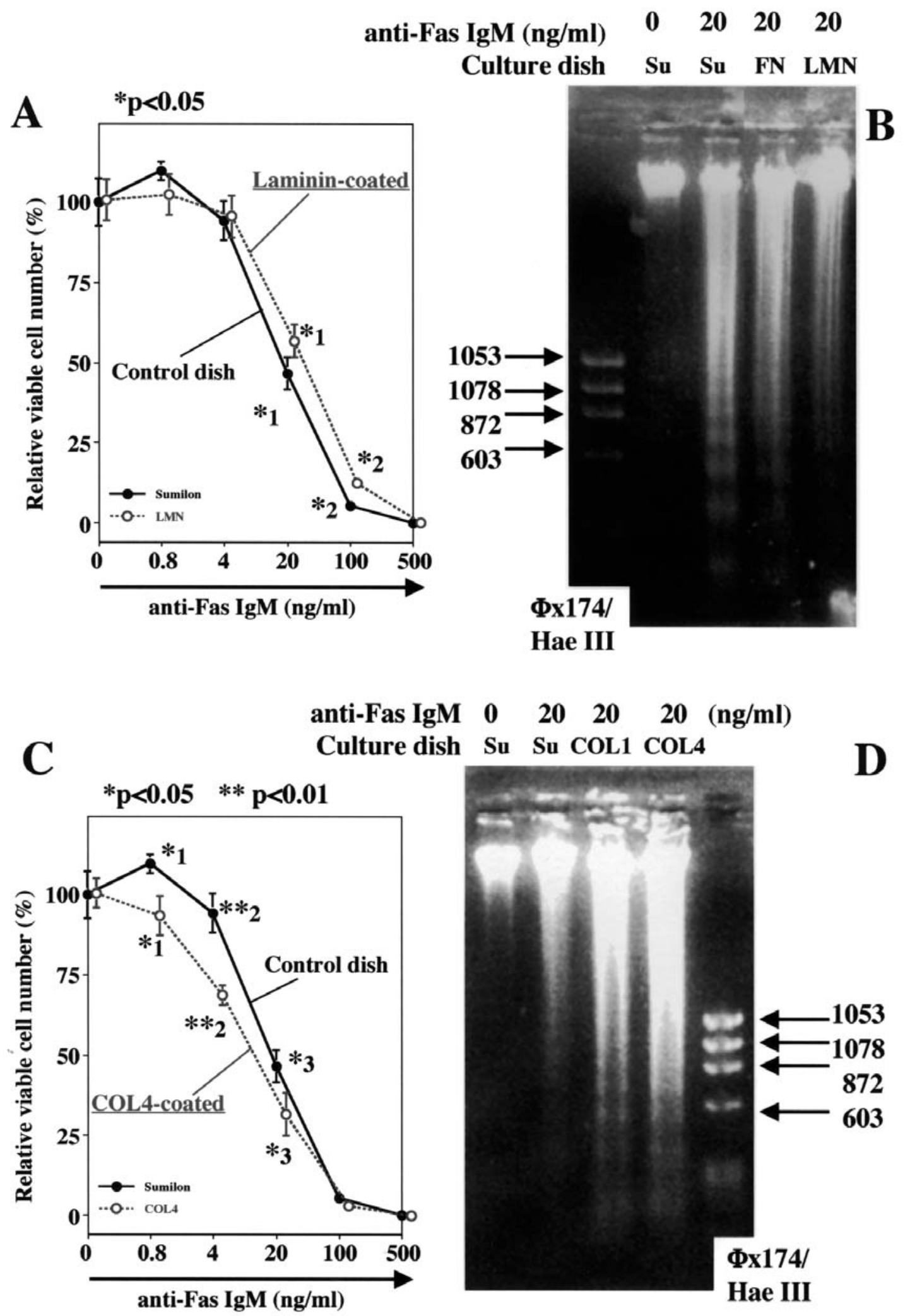

Figure 5. Effects of ECM components on Fas-mediated apoptosis of HHUA cells. All experiments were performed simultaneously. (A) Laminin significantly inhibited Fas-mediated growth inhibition of HHUA cells. The closed circles and solid line represent the growth inhibition curve for the control cells cultured on a control 96-well culture plate (Nalge Nunc International). The open circles and dotted line represent the growth inhibition curve for cells cultured on a laminin-coated culture plate. ${ }^{*} 1$ and ${ }^{*} 2$ show significant differences. ${ }^{*} 1$ and ${ }^{*} 2, p<0.05$. (B) Laminin inhibited Fas-mediated DNA fragmentation in HHUA cells. Su, total DNA from the cells cultured in a control culture dish (Sumitomo, Tokyo, Japan); FN, total DNA from the cells cultured in a fibronectin-coated dish; LMN, total DNA from the cells cultured in a laminin-coated dish. (C) COL-4 significantly enhanced Fas-mediated growth inhibition of HHUA cells. The closed circles and solid line represent the growth inhibition curve for the control cells cultured on a control 96-well culture plate (Nalge Nunc International). The open circles and dotted line represent the growth inhibition curve for the cells cultured on a COL-4-coated culture plate. ${ }^{*} 1,{ }^{* *} 2$ and ${ }^{*} 3$ show significant differences. ${ }^{*} 1$ and ${ }^{*} 3, \mathrm{p}<0.05 .{ }^{* *} 2, \mathrm{p}<0.01$. (D) COL-4 enhanced Fas-mediated DNA fragmentation in HHUA cells. Su, total DNA from the cells cultured in a control culture dish (Sumitomo); COL1, total DNA from the cells cultured in a type I collagen-coated dish; LMN, total DNA from the cells cultured in a COL-4-coated dish. 
and ECM components affects cell proliferation, cell colony structure and Fas-mediated apoptosis. This means that, during the proliferative phase, the effects of laminin are stronger than those of COL-4, because COL-4 expression is significantly decreased at that time. In the late proliferative period, laminin enhances endometrial epithelial cell growth and inhibits epithelial apoptosis, thus stimulating endometrial epithelial regeneration after menstrual shedding. During the secretory phase, the effects of COL-4 become dominant, because laminin expression in luminal surface BM-like-S is significantly decreased. During this phase, COL-4 may inhibit the proliferation of the luminal surface epithelium where an embryo often implants, and may enhance Fas-mediated apoptosis, which is thought to be involved in implantation $(24,25)$, thus increasing the embryo-receptivity. Laminin and COL-4 located in BM-like-S in the normal human endometrial epithelium are able to regulate not only the skeletal structure of the endometrium, but also menstrual cycle-dependent endometrial epithelial remodeling.

\section{Acknowledgements}

This study was supported in part by a grant-in-aid for scientific research from the Ministry of Education, Science, Sports and Culture of Japan.

\section{References}

1. Bilalis DA, Klentzeris LD and Fleming S: Immunohistochemical localization of extracellular matrix proteins in luteal phase endometrium of fertile and infertile patients. Hum Reprod 11: 2713-2718, 1996.

2. Aplin JD, Charlton AK and Ayad S: An immunohistochemical study of human endometrial extracellular matrix during the menstrual cycle and first trimester of pregnancy. Cell Tissue Res 253: 231-240, 1988.

3. Wewer UM, Damjanov A, Weiss J, Liotta LA and Damjanov I: Mouse endometrial stromal cells produce basement-membrane components. Differentiation 32: 49-58, 1986.

4. Iwahashi M, Muragaki Y, Ooshima A and Nakano R: Decreased type IV collagen expression by human decidual tissues in spontaneous abortion. J Clin Endocrinol Metab 81: 2925-2929, 1996.

5. Tanaka T, Wang C and Umesaki N: Autocrine/paracrine regulation of human endometrial stromal remodeling by laminin and type IV collagen. Int J Mol Med 22: 581-587, 2008.

6. Brar AK, Frank GR, Richards RG, et al: Laminin decreases PRL and IGFBP-1 expression during in vitro decidualization of human endometrial stromal cells. J Cell Phys 163: 30-37, 1995.

7. Mizuno K, Tanaka T, Umesaki N and Ogita S: Inhibition of cAMP-mediated decidualization in human endometrial stromal cells by IL-1ß and laminin. Horm Metab Res 31: 307-310, 1999.

8. Puistola U, Roennberg L, Martikainen H and TurpeenniemiHujanen T: The human embryo produces basement membrane collagen (type IV collagen)-degrading protease activity. Hum Reprod 4: 309-311, 1989

9. Autio-Harmainen H, Sandberg M, Pihlajaniemi T and Vuorio E: Synthesis of laminin and type IV collagen by trophoblastic cells and fibroblastic stromal cells in the early human placenta. Lab Invest 64: 483-491, 1991.
10. Romagnano L and Babiarz B: The role of murine cell surface paractisyltransferase in trophoblast: ly trophoblastic cells and fibroblastic stromal cells in the early human placenta. Lab Invest 64: 483-4aminin interactions in vitro. Dev Biol 141: 254-261, 1990.

11. Qin L, Wang YL, Bai SX, et al: Temporal and spatial expression of integrins and their extracellular matrix ligands at the maternal-fetal interface in the rhesus monkey during pregnancy. Biol Reprod 69: 563-571, 2003.

12. Park CC, Zhang H, Pallavicini M, et al: Beta1 integrin inhibitory antibody induces apoptosis of breast cancer cells, inhibits growth, and distinguishes malignant from normal phenotype in three dimensional cultures and in vivo. Cancer Res 66: 1526-1535, 2006.

13. Zhao X, Wang D, Zhao Z, et al: Caspase-3-dependent activation of calcium-independent phospholipase A2 enhances cell migration in non-apoptotic ovarian cancer cells. J Biol Chem 281: 29357-29368, 2006.

14. Edick MJ, Tesfay L, Lamb LE, et al: Inhibition of integrinmediated crosstalk with epidermal growth factor receptor/Erk or Src signaling pathways in autophagic prostate epithelial cells induces caspase-independent death. Mol Biol Cell 18: 2481-2490, 2007.

15. Edderkaoui M, Hong P, Lee JK, et al: Insulin-like growth factor-I receptor mediates the prosurvival effect of fibronectin. $J$ Biol Chem 282: 26646-26655, 2007.

16. Tanaka $\mathrm{T}$ and Umesaki $\mathrm{N}$ : Cytokine regulation of apoptotic susceptibility in a human endometrial epithelial cell line. J Reprod Immunol 47: 105-119, 2000.

17. Ishiwata I, Ishiwata C, Soma M, et al: Establishment of human endometrial adenocarcinoma cell line containing estradiol-17ß and progesterone. Gynecol Oncol 17: 281-290, 1084.

18. Mikami M, Sakai K, Yoshiki J, et al: Differentiation of endometrial carcinoma cell lines cultured in type 1 collagen gel and significance of sulfate lipid. Acta Obstet Gynecol Jpn 48: S348, 1996 .

19. Tanaka T and Umesaki N: Fas antigen (CD95) mediates cell survival signals to regulate functional cellular subpopulations in normal human endometrial stromal cells. Int J Mol Med 11: 757-762, 2003.

20. Futter CE, Crowston JG and Allan BD: Interaction with collagen IV protects lens epithelial cells from Fas-dependent apoptosis by stimulating the production of soluble survival factors. Invest Ophthalmol Vis Sci 46: 3256-3262, 2005.

21. Gendron S, Couture J and Aoudjit F: Integrin alpha2beta1 inhibits Fas-mediated apoptosis in $\mathrm{T}$ lymphocytes by protein phosphatase 2A-dependent activation of the MAPK/ERK pathway. J Biol Chem 278: 48633-48643, 2003

22. Tanaka Y, Nakayamada S, Fujimoto H, Okada Y, Umehara H, Kataoka T and Minami Y: H-Ras/mitogen-activated protein kinase pathway inhibits integrin-mediated adhesion and induces apoptosis in osteoblasts. J Biol Chem 277: 21446-21452, 2002.

23. Estrugo D, Fischer A, Hess F, Scherthan H, Belka C and Cordes N: Ligand bound beta1 integrins inhibit procaspase- 8 for mediating cell adhesion-mediated drug and radiation resistance in human leukemia cells. PLoS ONE 2: e269, 2007.

24. Kamijo T, Rajabi MR, Mizunuma H, et al: Biochemical evidence for autocrine/paracrine regulation of apoptosis in cultured uterine epithelial cells during mouse embryo implantantion in vitro. Mol Hum Reprod 4: 990-993, 1998.

25. Galn A, O'Connor JE, Valbuena D, et al: The human blastocyst regulates endometrial epithelial apoptosis in embryonic adhesion. Biol Reprod 63: 430-439, 2000. 